\title{
Status Report on the Superconducting Dipole Magnet Production for the $\mathrm{LHC}$
}

\author{
M. Bajko, F. Bertinelli, B. Bellesia, P. Fessia, P. Hagen, J. P. Koutchouk, J. Miles, M. Modena, M. Pojer, \\ L. Rossi, G. de Rijk, F. Savary, E. Todesco, D. Tommasini, J. Vlogaert, C. Vollinger, and E. Wildner
}

\begin{abstract}
In August 2006, about $95 \%$ of the production of the 1232 LHC superconducting dipole cold masses, whose coils are wound with $\mathrm{Cu} / \mathrm{Nb}$-Ti cables, is completed. One of the 3 manufacturers, having produced one third of the required magnets, completed its production in the end of 2005 . The acceptance of the magnets takes place after the $1.9 \mathrm{~K}$ performance tests and has been issued for more then $\mathbf{1 0 0 0}$ magnets so far. More then half of the dipole magnets are already installed in the tunnel. The paper reviews the main features of the dipoles, the most important steps of the manufacturing and the most critical operations. The quality control and the critical non-conformities that have led, for instance, to a swift campaign of investigations and repairs of few subcomponents (diode assembly, cold bore tube to welding flare fillet weld) are discussed. The status of the production and the performance of the tested dipoles will be presented. Finally the expected schedule for the completion of the production will be shown.
\end{abstract}

Index Terms-Dipole, LHC, magnets, $\mathrm{Nb}$-Ti, superconductivity.

\section{INTRODUCTION}

$\mathbf{T}$ HE Large Hadron Collider (LHC) [1] is designed to accelerate two counter rotating proton beams up to a top energy of $7 \mathrm{TeV}$. The main dipole magnets, called also main bends (MBs) for the LHC, filling more then $2 / 3$ of the tunnel, are made with coils wound with Rutherford type cable, based on $\mathrm{Cu}$ stabilized $\mathrm{Nb}-\mathrm{Ti}$ superconductor wires. The $27 \mathrm{~km}$ long ring needs $1232 \mathrm{MBs}$ providing a maximum field of $9 \mathrm{~T}$. A contract, for the production of 1248 dipole magnets, was placed in autumn 1999 with 3 European companies: consortium AlstomJeumont in France, ASG Superconductors in Italy and Babcock Noell Nuclear in Germany. In August 2006 the production is well advanced: about $95 \%$ of the required magnets were produced and delivered to CERN and more then half of them, after having passed all acceptance tests, are installed in the LHC tunnel (Fig. 1).

\section{MAin FeATURES of THE MBs}

The LHC MBs are designed to work in pressurized superfluid helium at $1.9 \mathrm{~K}$. This very low temperature allows to attain a higher central field, corresponding to approximately $20 \%$ increase in beam energy w.r.t the one obtained at $4.2 \mathrm{~K}$. The MBs

Manuscript received September 2006. This work was supported in part by the AT Department of CERN.

M. Bajko is with CERN, 1211 Geneva 23, Switzerland (e-mail: marta.bajko@cern.ch).

Color versions of one or more of the figures in this paper are available online at http://ieeexplore.ieee.org.

Digital Object Identifier 10.1109/TASC.2007.899697

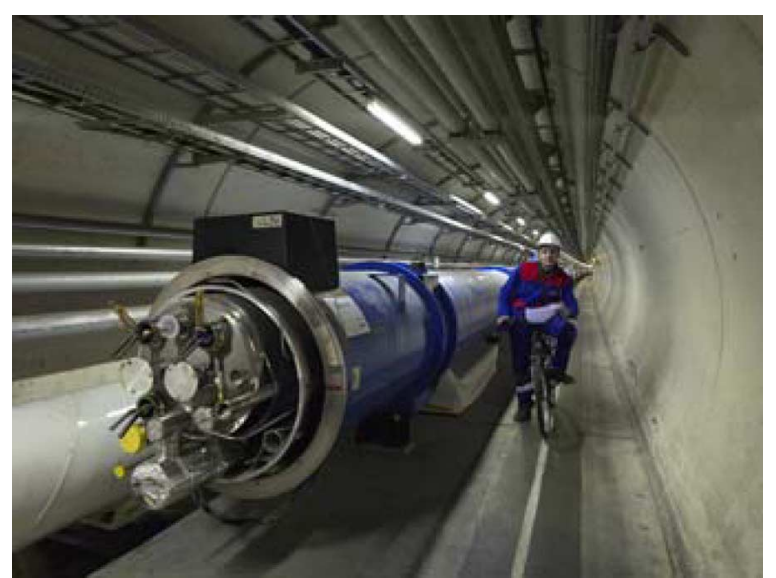

Fig. 1. View of the LHC ring. The 17th July 2006 the 616th dipole is installed.

are designed for a nominal operational current of $11850 \mathrm{~A}$, generating a central dipole field of $8.33 \mathrm{~T}$, with a possibility to reach up to $9 \mathrm{~T}$ also referred here after to as "ultimate field".

\section{A. The Superconducting Cable. The Layout of the Coils}

The transverse cross-section of the coils for the LHC dipoles consist of two layers with different cables.

The non-copper critical current density in the inner cable is $1480 \mathrm{~A} / \mathrm{mm}^{2}$ at $10 \mathrm{~T}$, while for the outer cable is $2000 \mathrm{~A} / \mathrm{mm}^{2}$ at $9 \mathrm{~T}$, both at $1.9 \mathrm{~K}$ temperature [1]. The cables are insulated with polyimide tape of $50 \mu \mathrm{m}$ thickness in 3 layers. The six-block arrangement in two layers increases the stability and leaves more room for minor optimizations. Copper wedges ensure the spacing of the blocks in each layer. The precise azimuthal and radial position of the blocks is essential to achieve the nominal magnetic field with its designed accuracy in the beam channel. In order to avoid the sorting during the production, it was required that each layer be produced such to be dimensionally identical within $100 \mu \mathrm{m}$. The variation of $100 \mu \mathrm{m}$ in the azimuthal direction corresponds to a variation of $0.1 \%$ of the main field and, up to 3.5 units of the sextupolar harmonics, and about $12 \mathrm{MPa}$ of azimuthal pre-stress of the coils.

\section{B. The Collared Coils}

Each pole is composed of an outer and of an inner layer. They are connected electrically in series by soldering the two cables with a high conductivity copper, to improve the thermal stability and the mechanical rigidity of the junction.

When a quench occurs in the magnet the so-called quench heaters $(\mathrm{QH}), 8$ per magnet, warm the entire outer layer to 
rapidly raise the temperature to above $10 \mathrm{~K}$ in about $20 \mathrm{~ms}$, thus forcing transition from superconducting to resistive state over the whole coil, protecting the magnet from local damage.

The $\mathrm{QH}$ consist of partially copper plated stainless steel strips of about $25 \mu \mathrm{m}$ thickness. The strips are laminated inside a polyimide insulating film and are placed on the outer layer of each coil. For the protection of the magnets 4 out of the 8 circuits are needed, those placed in the high-field region. The other 4 circuits are used only in case of defect in the normally connected circuits.

Ground insulation, composed of 4 layers of polyimide tapes of $125 \mu \mathrm{m}$, is required between the coils and the stainless steel collars. Beside its electrical function of insulating the coils, adding an extra layer between the poles and coils also provides for adjusting the magnetic field.

The collars are twin, i.e. common for the two dipoles. They are obtained by fine blanking $3 \mathrm{~mm}$ thick austenitic steel sheets [2]. The shape of the collars has been designed such to take into account and to partially compensate the mechanical deformations of the cavity due to the residual pre-stress after collaring and cool down.

\section{The Cold Mass}

The magnetic circuit surrounds the collared coils; the whole is contained in a shrinking cylinder and closed by end covers, which constitute the helium vessel. The yoke consists of precision punched laminations of $5.7 \mathrm{~mm}$ in thickness assembled in packs of up to $1.5 \mathrm{~m}$ length with a filling factor of $98 \%$. The laminations are made of magnetic steel with low carbon content to obtain the maximum magnetic permeability also at low and intermediate field. The He heat exchanger tube carries away the heat during the cooling down from $4.2 \mathrm{~K}$ to $1.9 \mathrm{~K}$ and during operation at $1.9 \mathrm{~K}$. In operation it carries saturated superfluid helium at $1.6 \mathrm{kPa}$. A seamless round oxygen-free copper tube, equipped with seamless austenitic stainless steel sleeves on both ends, composes the heat exchanger tube [1]. The main parts of the helium vessel, that contains in operation at $1.9 \mathrm{~K}$ a static bath of superfluid $\mathrm{He}$ at atmospheric pressure, are: the shrinking cylinder composed of two half shells and the two end covers (made of AISI $316 \mathrm{LN}$ steel grade) and the external auxiliary bus bar tube. The shrinking cylinder gives the necessary rigidity to the cold mass and provides the inertia needed to maintain the geometry of the cold mass. The end covers have openings for the helium heat exchanger, the cold bore tubes, the bus bars and diode container. Most of the elements necessary for the interconnection, such as flexible bellows and flanges, are fixed by welding to the end covers. The cold bore tubes (CBT) provide the passage for the circulating particle beams: the wall of the non-magnetic austenitic stainless steel tube separates the helium volume from the beam vacuum. The tube is insulated electrically on their outer wall with epoxy coated polyimide tape. An essential element for the protection of the MBs is the diode mounted inside the helium vessel, which is working at $1.9 \mathrm{~K}$. When a quench occurs, an initial current of up to $13 \mathrm{kA}$, which decays exponentially with a time constant of $100 \mathrm{~s}$, flows through the diode bypassing the magnet.

\section{The Most Important Assembly StePs}

\section{A. Pole Assembly}

For the winding of the coils, the positioning of the blocks is the most important operation with regards to the field quality. The operation requires not only a reliable winding machine, designed for that purpose, but also very experienced operators. Once the coils are wound, they are polymerized, under a maximum pressure of 80-90 MPa, in order to keep the turns and cable blocks in place during and until the collaring operation is done. The tolerance range of the polymerization temperature is $\pm 3^{\circ} \mathrm{C}$, to obtain a good gluing homogeneity of the coils. The coils are then measured under pressure and their eventual geometrical errors are corrected with shims. A good control of the coils size allowed applying over nearly the whole production the same nominal shims. In order to have along the coils a good structural homogeneity, the ends of the coils, made of impregnated glass fiber, are measured separately and are shimmed individually. The measurements, under a dedicated press with the goal to determine the right shim dimension as a function of a desired pressure distribution, also made it possible to detect short circuits due to insulation errors of the cable.

A very delicate operation is the splice soldering, done with $130 \mathrm{~mm}$ overlap of the two types of cables. The resistance of the junction should be of the order of $0.5 \mathrm{n} \Omega$ at $4.2 \mathrm{~K}$. The difficulty of the operation consists also in the quality control, as it is limited to dimensional and to electrical resistance measurements at room temperature. Although such tests cannot provide complete confidence regarding operation at $1.9 \mathrm{~K}$, in only one case has quench performance been found to be affected.

The most critical part of the pole construction is the assembly of the quench heaters with the coils. In particular, the quench heaters must be carefully supported during the collaring to avoid mechanical damage of the metallic strips.

\section{B. Collaring}

The collaring is done under a dedicated press where the pressure is increased step by step until the locking roads can be inserted into the stack. At each step, tests are performed to check the electrical integrity of the coils and the insulation. A fraction of insulation problems are detected at this stage. Further investigations regarding the location of short circuits have been done on the collared coils with warm magnetic measurements and subsequent inverse calculation of the measured harmonics [3]. This method is an excellent tool not only for detection of shorts, but also for other assembly errors, such as cable or block displacement with respect to its nominal position or the presence, due to human error, of a double coil protection sheet. The magnetic measurements are performed on $100 \%$ of the collared coils and repeated for all magnets at the very end of the cold mass assembly [4].

The $\mathrm{QH}$ are connected in series and the joints are inserted in a special groove in the coil heads. The integrity is checked, at the end of the collaring, by a test consisting of a discharge into the heater strip of the energy of a capacitor bank that provides a peak current of $75 \mathrm{~A}$. This test is also repeated at the end of the cold mass assembly and at the test bench once the magnets are ready 
for the cold test, completed by the measurements of the electrical resistance of every single circuit of quench heaters and are compared the results between the different steps of assembly. A defect (partial discontinuity) of the quench heater strips was detected with such test in nearly $2 \%$ of the magnets produced by one of the 3 companies. The test can damage the coil in contact with the incriminated strip. Due to such a defect of the quench heater, one pole was destroyed during powering of a magnet at $1.9 \mathrm{~K}$.

\section{Cold Mass Assembly}

The yoke assembly consist in mounting the pre-assembled lamination packs in two half yokes. The two half shells are welded together in a purposed built welding press with the goal to build up a circumferential stress of $150 \mathrm{MPa}$ at room temperature. The welding procedure is a combination between Surface Tension Transfer (STT) procedure and a pulsed MIG process. To obtain the necessary quality of the weld, required by the European pressure vessel regulations in force for, the welding parameters were optimized in closed collaboration with the supplier of the welding system. $100 \%$ of the welds were checked by $\mathrm{X}$-Ray on the pre-series magnets (30 by each company). Then the rate of checks was gradually reduced to $10 \%$. Visual checks and destructive test on samples were also performed.

\section{Bending the Cold Mass. Final Assembly. Welds}

The magnets are curved with a sagitta of $9.14 \mathrm{~mm}$ over a chord of $14343 \mathrm{~mm}$, corresponding to the magnetic length of the MBs at room temperature. To minimize the spring back, which occurs when the magnet is released from the welding press, the half cylinders are pre-curved at the manufacturing stage. In spite of the difficulties encountered at the beginning of the production to obtain the curvature of the magnets within the required tolerances of $\pm 1 \mathrm{~mm}$, once the welding press shape was fixed to approximately $12 \mathrm{~mm}$, only a very few magnets were accepted with a non-conforming curvature. On the other hand, already at the beginning of the project, instability was detected in the curvature of the magnets. Blocking the central foot on which the magnets stays inside the cryostat solved this problem. Unfortunately, the structural strength of the central foot does not allow adjusting the shape of the magnets to its nominal position [5]. Changing of the design of the central foot at this stage of the project was not anymore possible (See Section IV-B how this has been handled).

For every welded joint the welding procedure and the welder have been qualified before the production started. In spite of that and following a visual inspection made on an important number of magnets already delivered to CERN, about $250 \mathrm{X}$-Ray checks were made and the conformity of welds checked with respect to the quality level "B" as per the international standard EN 25817. Given the type of indication and its correlation with surface defects visible inside the cold bore tube, a repair of the non conforming welds was proposed to guarantee the long term mechanical strength and leak tightness of the cold mass envelope. The difficulty of the above mentioned weld consists in achieving on the cold bore tube side a minimum of $33 \%$ and a maximum of $66 \%$ penetration into the wall of the $1.5 \mathrm{~mm}$ thick cold bore tube.
The extent of these non-conformities triggered a review of the acceptance criteria. The loading case of this weld has been estimated, evaluating the possible movements between the cold mass and the cold bore tube. Differential movement could originate from the mechanical loads applied to the cold mass, mainly due to the gas pressure after a quench or to the electromagnetic force and temperature gradients.

The most severe condition is the case of the electromagnetic forces at $9 \mathrm{~T}$, which give rise to a relative displacement of the two welded parts up to $1.2 \mathrm{~mm}$. 3D finite element modeling was done to evaluate the fatigue during the life time of the LHC, estimated to be 10000 current cycles. One cold mass presenting severe non-conformity on that weld was submitted to a dedicated test. The objective was to submit the magnet to 5000 cycles between $0.55-8.4 \mathrm{~T}$. To shorten the test to 4000 cycles the magnet was cycled from $1.4 \mathrm{~T}$ to $8.82 \mathrm{~T}$. Before and after the test faulty weld was X-Rayed. The magnet maintained its leak tightness and no evolution of the cracks were seen on the X-Rays. This study allowed us to reduce the number of repairs and to establish for all non-conform welds a repair procedure and new detailed acceptance criteria. Repairs were made at CERN on 16 magnets. The quality control in the industry was reinforced regarding particularly that weld [6].

The diode stack installation is one of the last assembly operations to be done. The diode box is part of the pressure vessel. The assembly procedure has been revised at the end of the pre-series magnets following the observation of weak points concerning the diode to bus bar connection. A too high contact resistance value may result in excessive heating and irreversible damage to the diode stack, with as a possible consequence the destruction of the cold mass. A typical electrical resistance value of the before mentioned connection should be about $1 \mu \Omega$ without exceeding $2 \mu \Omega$ [7]. Due to a high resistance measured on the connection $20 \%$ of the series magnets has been repaired at CERN.

\section{Quality of the Magnets}

\section{A. Magnetic Field Quality}

The measurements of the magnetic field at room temperature have been used to steer the production towards the beam dynamics targets, using the warm-cold correlation established at the beginning of the production. Following two changes implemented in the cross-section, today, with $95 \%$ of magnets produced, both the average and the spread of the transfer function and of field harmonics are within the targets [8].

\section{B. Geometry}

The main geometrical parameters relevant for the beam are the shape of the cold mass and the position of the corrector magnets mounted inside the cold mass. On top of the tolerances imposed to the magnet assemblers, the magnets have been put in 3 main classes: golden, silver and mid-cell. The golden magnets are those having an excellent shape with a significant margin for the aperture. They can be placed at any position in the LHC machine. The mid-cell magnets are those exceeding the tolerance of $\pm 1.5 \mathrm{~mm}$ in the horizontal plane and of $\pm 0.8 \mathrm{~mm}$ in the vertical plane of up to $0.5 \mathrm{~mm}$. Those magnets can be placed only in the middle of a cell, where aperture requirements are 


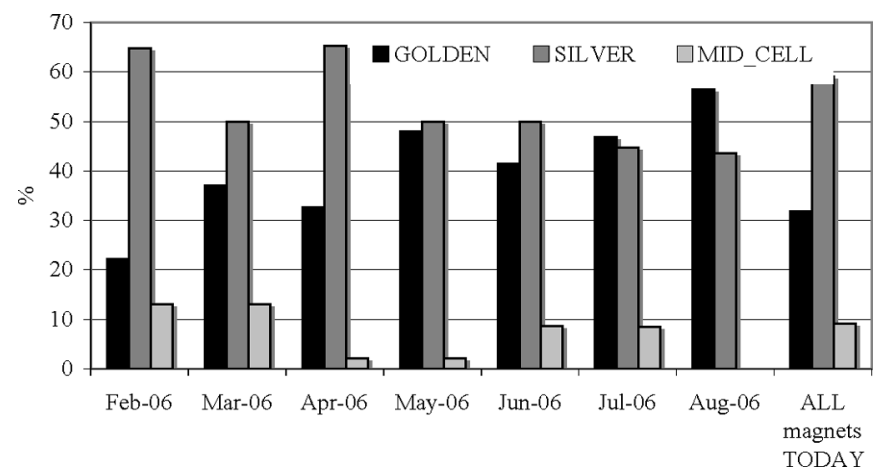

Fig. 2. Evolution of the magnet classes in the production in 2006 .

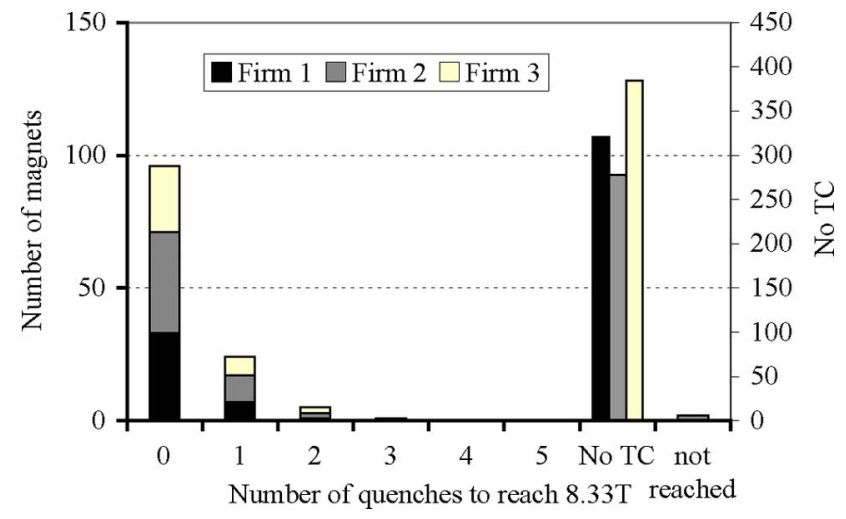

Fig. 3. Histogram of the number of quenches to reach $8.33 \mathrm{~T}$ after TC.

much less stringent [9]. In industry more then $30 \%$ of the magnets produced were in the golden class where we need at least $13 \%$ and less then $9 \%$ are in the mid-cell class limited to $10 \%$ (see Fig. 2).

All geometrical parameters with tight tolerances (of the order of magnitude of $0.1 \mathrm{~mm}$ ) coming from requirements of the interconnections of the magnets in the LHC tunnel, were produced by the companies, fully within the tolerances.

\section{Quench Performance and Electrical Integrity}

More than $38 \%$ of the magnets have reached the nominal field without any training quench during the power test at cold. $12 \%$ of the magnets did not reached the ultimate field in 9 ramps. For such magnets, a second run, following a thermal cycle (TC), was made (See Fig. 3).

Of these magnets, $77 \%$ reached the nominal field with a single training quench. For the remaining magnets the quench performance was considered insufficient.

About $2.5 \%$ of dipoles were not respecting the acceptance criteria after cold test and were returned to the factories for repairs. On 11 magnets a default was detected in the QHs causing in most of the cases an insulation defect and/or a short circuit between $\mathrm{QH}$ and coil. Because of insufficient quench performance, 14 magnets have been rejected and sent back to the companies

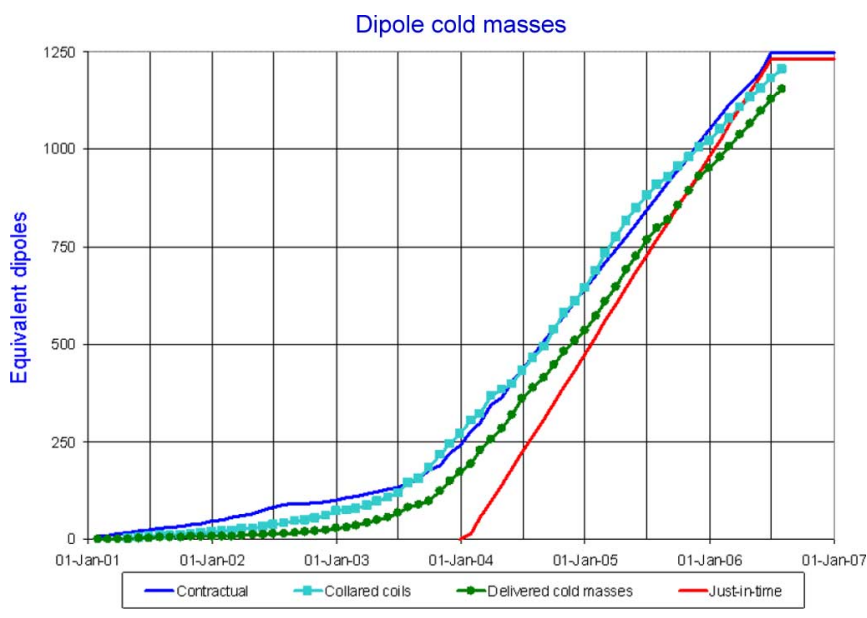

Fig. 4. LHC dashboard: Advancement of the production. The actual deadline has been moved to 30th of October 2006.

for repairs. 12 of them have already been repaired and all of them accepted after cold test.

\section{The Expected Delivery Schedule}

The dipole magnet production is advancing well as shown in Fig. 4. The completion of the production for the tunnel (1232) is foreseen for the end of October 2006 in agreement with the present LHC master schedule, which requires all magnets to be cold tested by end of 2006. The production of the 45 spares is foreseen by February 2007.

\section{REFERENCES}

[1] O. Bruning et al., "LHC Design Report," vol. I, The LHC Main Ring, CERN-2004-003, June 4, 2004.

[2] M. Bajko, P. Fessia, and D. Perini, "Statistical studies of the robustness of the LHC main dipole mechanical structure," IEEE Trans. Appl. Supercond., vol. 10, no. 1, pp. 77-80, 2000.

[3] B. Bellesia, G. Molinari, C. Santoni, W. Scandale, and E. Todesco, "Short circuit localization in the LHC main dipole coils by mean of room temperature magnetic measurements," IEEE Trans. Appl. Supercond., vol. 16, no. 2, pp. 208-211, 2006.

[4] C. Vollinger and E. Todesco, "Identification of assembly faults through the detection of magnetic field anomalies in the production of the LHC dipoles," IEEE Trans. Appl. Supercond., vol. 16, no. 2, pp. 204-207, 2006.

[5] M. Bajko et al., Executive Summary of the Workshop on LHC Dipole Geometry and Stability and Preparation for the Implementation of Changes, CERN EDMS 568828, 2004.

[6] P. Fessia and F. Savary, Acceptance Criteria for the Cold Bore Tube to Wedling Flare Fillet Weld of the Main Superconducting Dipoles for LHC in the Frame of the Repair Activities, CERN EDMS 677406, Nov. 2005.

[7] M. Cornelis, Intsallation of the Protection Diode Stack for the LHC Main Dipoles, CERN EDMS 568828, March 2004.

[8] E. Todesco, B. Bellesia, P. Hagen, and C. Vollinger, "Trends in filed quality along the production of the LHC dipoles and differences among manufacturers," IEEE Trans. Appl. Supercond., vol. 16, no. 2, pp. 419-424, 2006.

[9] S. Fartoukh, "Classification of the LHC main dipole and installation startegy," in Proc of LHC Workshop Chamonix-XIII, 2004, pp. $148-158$. 